\title{
Dipole and quadrupole polarizabilities and shielding factors of beryllium from exponentially correlated Gaussian functions
}

\author{
Jacek Komasa* \\ Quantum Chemistry Group, Department of Chemistry, A. Mickiewicz University, Grunwaldzka 6, 60-780 Poznań, Poland
}

(Received 25 July 2001; published 14 December 2001)

\begin{abstract}
Dynamic dipole and quadrupole polarizabilities as well as shielding factors of the beryllium atom in the ground state were computed at real frequencies by using the variation-perturbation method. The zeroth- and the first-order wave functions were expanded in many-electron basis of exponentially correlated Gaussian functions. The 1600-term expansion of the unperturbed wave function yielded the ground-state energy accurate to $1 \mathrm{~cm}^{-1}$. The first-order wave functions were expanded in very large bases (4800 and $4400 \mathrm{terms}$ ). The nonlinear parameters of the first-order correction functions were optimized with respect to both the static and dynamic polarizabilities, and with respect to the excited-state energies. The procedure employed ensures a high accuracy of determination of dynamic properties in a wide range of frequencies and correct positions of the transition poles. Test calculations, performed on $\mathrm{He}$ and $\mathrm{Li}$, confirmed the ability of this method to obtain the atomic properties with very high accuracy. The final values of the static properties of Be were $37.755 e^{2} a_{0}^{2} E_{H}^{-1}$ and $300.96 e^{2} a_{0}^{4} E_{H}^{-1}$ for the dipole and quadrupole polarizabilities, respectively, and 1.4769 for the quadrupole shielding factor. The convergence of the atomic properties with the size of the expansion of both the zerothand first-order functions was checked. Thanks to very high accuracy of the unperturbed wave function and the efficient method of construction of the first-order wave functions, the dynamic polarizability results presented in this work are of benchmark quality. As a by-product of this project, a set of the most accurate upper bounds to the energies of ${ }^{1} P$ and ${ }^{1} D$ states of Be was obtained.
\end{abstract}

DOI: 10.1103/PhysRevA.65.012506

PACS number(s): 32.10.Dk, 31.25.-v

\section{INTRODUCTION}

One of the most important aspects of our knowledge of many-electron systems is the ability to predict their behavior in external fields. Particular attention of theoreticians is drawn to the polarizabilities, which can be relatively easily modeled mathematically and are involved in a variety of physical phenomena. These response properties are often linked to the optical properties of matter, scattering processes or interatomic interactions. In particular, the frequencydependent polarizabilities enter the formulas defining second refractive virial coefficient, Verdet constant, van der Waals coefficients, refractive index, etc. A growing interest in the accurate knowledge of polarizability of atomic gases is observed. Such theoretically predicted polarizability, if sufficiently accurate, might serve to calibrate measuring apparatus for various experiments [1,2] and to independently estimate fundamental constants of physics and chemistry [3-6].

The dipole polarizability of an atom $\left(\alpha_{1}\right)$ corresponds to a dipole moment induced in the atom interacting with an external electric field $\left(F_{1}\right)$. Similarly, quadrupole polarizability $\left(\alpha_{2}\right)$ is related to a quadrupole moment induced by an external electric field gradient $\left(F_{2}\right)$. There are two other quantities closely related to the polarizabilities, namely, the dipole $\left(\gamma_{1}\right)$ and quadrupole $\left(\gamma_{2}\right)$ shielding factors. They give a picture of dipole and quadrupole moments induced in the electron charge distribution by pertinent nuclear moments. Alternatively, $\gamma_{1}$ and $\gamma_{2}$ can be treated as parameters describing

\footnotetext{
*Electronic address: komasa@man.poznan.pl
}

the change in the field and field gradient, respectively, experienced by the nucleus, resulting from the electron cloud shielding $\left(\gamma_{\lambda}>0\right)$ or antishielding $\left(\gamma_{\lambda}<0\right)[7]$. Although the physical nature of the shielding factors slightly differs from that of polarizabilities, they are mathematically closely coupled and in this work they are studied together.

For the beryllium atom the values of these properties have not been experimentally determined yet and we have to rely on the theoretical predictions. In cases like this, it is crucial to have an access to reliable reference values. The aim of this study was to supply such benchmark values of the dynamic dipole and quadrupole polarizabilities and shielding factors. For an accurate description of polarizability, the electron correlation has to be taken into consideration at a very high level. Additionally, good description of the outer, energetically less important, region of the electron density distribution is indispensable. These requirements are met by very flexible, explicitly correlated wave functions employed in this paper.

\section{METHOD}

The dipole and quadrupole polarizabilities appear as expansion coefficients in the expression for the energy change caused by an electric field and electric field gradient [8]. Formally, the polarizability is defined as a second derivative of the perturbation-dependent energy [9]

$$
\alpha_{\lambda}=-\left(\frac{\partial^{2} E\left(F_{\lambda}\right)}{\partial F_{\lambda}^{2}}\right)_{F_{\lambda} \rightarrow 0}
$$

and can be related to the second-order perturbation energy by 


$$
\alpha_{\lambda}=-2 E_{\lambda}^{(2)}
$$

In the notation incorporated hereinafter, $\lambda=1$ for dipole and $\lambda=2$ for quadrupole properties.

We shall work in the nonrelativistic infinite nuclear mass framework. The mass polarization and the relativistic corrections $[5,6,10]$ to the dipole polarizability are the subject of our current study and will be presented separately. The atomic units are employed throughout this paper. In particular, $\hbar=1$ and the electron mass $m=1$ are assumed, $\alpha_{1}$ and $\alpha_{2}$ are expressed in units of $e^{2} a_{0}^{2} E_{H}^{-1}$ and $e^{2} a_{0}^{4} E_{H}^{-1}$, respectively, and the energy is expressed in the Hartree energy $\left(E_{H}\right)$. Both $\gamma_{1}$ and $\gamma_{2}$ are dimensionless.

If the total wave function $\Psi_{\lambda}=\Psi^{(0)}+F_{\lambda} \Psi_{\lambda}^{(1)}+\cdots$ (assumed real and normalized) satisfies the Hellmann-Feynman theorem [11-13], then $\alpha_{\lambda}$ and $\gamma_{\lambda}$ can be expressed conveniently as single integrals [14]:

$$
\begin{aligned}
& \alpha_{1}=-2 \int \Psi_{1}^{(1)}\left(\sum_{i=1}^{n} y_{i}\right) \Psi^{(0)} d \tau, \\
& \alpha_{2}=-4 \int \Psi_{2}^{(1)}\left(\sum_{i=1}^{n} y_{i} z_{i}\right) \Psi^{(0)} d \tau, \\
& \gamma_{1}=-2 \int \Psi_{1}^{(1)}\left(\sum_{i=1}^{n} \frac{y_{i}}{r_{i}^{3}}\right) \Psi^{(0)} d \tau, \\
& \gamma_{2}=-4 \int \Psi_{2}^{(1)}\left(\sum_{i=1}^{n} \frac{y_{i} z_{i}}{r_{i}^{5}}\right) \Psi^{(0)} d \tau .
\end{aligned}
$$

The Hellmann-Feynman theorem allows $\gamma_{1}$ to be determined a priori. For an $n$-electron atom with a nucleus of charge $Z$ $[14,15]$,

$$
\gamma_{1}=\frac{n}{Z}
$$

For this fact, $\gamma_{1}$ was recommended as a useful tool for assessment of the quality of approximated wave functions involved in Eqs. (2.3) and (2.5).

\section{A. The ansatz}

There are three different functions involved in the formulas (2.3)-(2.6). $\Psi^{(0)}$ is the unperturbed or zeroth-order wave function of the atom; $\Psi_{1}^{(1)}$ and $\Psi_{2}^{(1)}$ are the first-order correction functions resulting from the dipole and quadrupole perturbation, respectively. In this paper, all three functions are expressed in the form of antisymmetrized linear combinations of $n$-electron basis functions, $\psi_{k}^{(\kappa)}(\kappa=0$ or 1$)$,

$$
\widetilde{\Psi}^{(\kappa)}(\mathbf{r}, \boldsymbol{\sigma})=\hat{\mathcal{A}}\left(\Xi_{n, S, M_{S}}(\boldsymbol{\sigma}) \sum_{k=1}^{K^{(\kappa)}} c_{k}^{(\kappa)} \psi_{k}^{(\kappa)}(\mathbf{r})\right),
$$

where $\Xi_{n, S, M_{S}}(\boldsymbol{\sigma})$ is an $n$-electron spin function (e.g., $\Xi_{4,0,0}(\boldsymbol{\sigma})=\alpha \beta \alpha \beta-\beta \alpha \alpha \beta-\alpha \beta \beta \alpha+\beta \alpha \beta \alpha$ for the fourelectron singlet state), and where, in general, the linear coef- ficients $c_{k}^{(1)}$ of the expansion of the first-order function depend on the light frequency; $\mathbf{r}$ is a $3 n$-element vector of electron position coordinates and $\boldsymbol{\sigma}$ represents $n$ spin variables. The tilde over $\Psi$ is used to distinguish between the exact wave function and its approximation.

As the many-electron basis functions $\psi_{k}$, the exponentially correlated Gaussian (ECG) functions of Singer [16] are employed:

$$
\psi_{k}(\mathbf{r})=\Lambda_{m_{k}} \exp \left[-\left(\mathbf{r}-\mathbf{s}_{k}\right) \mathbf{A}_{k}\left(\mathbf{r}-\mathbf{s}_{k}\right)^{T}\right]
$$

with $\mathbf{s}_{k}$ restricted to zero- the natural choice for the position of the nucleus. The remaining nonlinear parameters are organized in the form of positive definite symmetric $n \times n$ matrices $\mathbf{A}_{k}$. $T$ superscript means a vector transposition. The preexponential factor $\Lambda_{m_{k}}=1$ for the unperturbed wave function, $\Lambda_{m_{k}}=y_{m_{k}}$ for $\widetilde{\Psi}_{1}^{(1)}$, and $\Lambda_{m_{k}}=y_{1} z_{m_{k}}$ for $\widetilde{\Psi}_{2}^{(1)}, y_{i}$, and $z_{i}$ are the Cartesian components of the $i$ th electron position vector. Such a choice of the basis functions ensures respectively $S, P$, and $D$ symmetry of the atomic wave functions. The $m_{k}$ subscript labels the electrons. An experience has shown that the restriction of $m_{k}$ to a single electron leads to erroneous convergence. In this work, all possible values $1 \leqslant m_{k} \leqslant n$ were used and spread out uniformly over all basis functions. Their presence makes possible using only a single spin function without loss of completeness.

The ECG wave functions have been proved to work very well for few-electron systems yielding in many cases the best variational energies available in the literature: $\mathrm{H}_{2}$ [17-19], $\mathrm{HeH}^{+}, \mathrm{H}_{3}^{+}$[18], $\mathrm{H}_{3}$ [20,21], $\mathrm{He}_{2}^{+}$, LiH [22], $\mathrm{He}_{2}$ [23,24], $\mathrm{HeHHe}^{+}[25], \mathrm{Be}[26,27], e^{+} \mathrm{LiH}$ [28]. Also many accurate expectation values of beryllium atom in position and momentum space come from the ECG calculations [24,29,30]. In the present paper, we extend this list by energies of a few lowest excited states and the second-order properties of Be.

\section{B. Variation-perturbation method}

For the harmonic, monochromatic perturbation of an angular frequency $\omega$, the stationary-state first-order functions are represented by [31]

$$
\begin{aligned}
\Psi_{\lambda}^{(1)}(\mathbf{r}, t)= & \Psi_{\lambda+}^{(1)}(\mathbf{r}) \exp \left[-i\left(E^{(0)}-\omega\right) t\right] \\
& +\Psi_{\lambda-}^{(1)}(\mathbf{r}) \exp \left[-i\left(E^{(0)}+\omega\right) t\right] .
\end{aligned}
$$

The plus and minus components, $\Psi_{\lambda \pm}^{(1)}$, can be obtained from the solution of the first-order perturbation equations (assuming $\Psi^{(0)}$ is known)

$$
\left(H^{(0)}-E^{(0)} \pm \omega\right) \Psi_{\lambda \pm}^{(1)}=-\hat{\mathcal{O}}_{\lambda} \Psi^{(0)},
$$

where $H^{(0)}$ and $E^{(0)}$ are the unperturbed Hamiltonian and energy, respectively, and $\hat{\mathcal{O}}_{1}=\sum_{i=1}^{n} y_{i}$ for the dipole and $\hat{\mathcal{O}}_{2}$ $=\sum_{i=1}^{n} y_{i} z_{i}$ for the quadrupole polarizability. Equations (2.11) can be solved variationally, i.e., by minimization of the Hylleraas functional [32,33], 


$$
\begin{aligned}
\mathcal{J}\left[\Psi_{\lambda \pm}^{(1)}\right]= & \int \Psi_{\lambda \pm}^{(1)}\left(H^{(0)}-E^{(0)} \pm \omega\right) \Psi_{\lambda \pm}^{(1)} d \tau \\
& +2 \int \Psi_{\lambda \pm}^{(1)} \hat{\mathcal{O}}_{\lambda} \Psi^{(0)} d \tau
\end{aligned}
$$

with respect to the parameters of the first-order function. $\Psi_{\lambda \pm}^{(1)}$ determined in this way enter the pertinent expressions for $\alpha_{\lambda_{ \pm}}$and $\gamma_{\lambda_{ \pm}}$, Eqs. (2.3)-(2.6). Finally, the frequencydependent polarizabilities and shielding factors are obtained from $\alpha_{\lambda}(\omega)=\alpha_{\lambda+}(\omega)+\alpha_{\lambda-}(\omega)$ and a similar equation for $\gamma_{\lambda}(\omega)$.

As shown by, e.g., Kolker and Michels [34], when $\Psi_{\lambda}^{(1)}$ is expanded in the complete set of the unperturbed Hamiltonian's eigenfunctions, one arrives at the spectral representation of $\alpha_{\lambda}(\omega)$. From this point of view, the first-order wave function involves an infinite number of excited states, including the continuum, so that it is not a trivial task to generate such a wave function with an accuracy comparable to those attainable for the unperturbed systems even if the wave function includes explicitly the electron correlation factor.

In the past, many accurate results were obtained within the variation-perturbation approach in connection with the explicitly correlated wave functions. For example, Glover and Weinhold [35] employed the Hylleraas-type wave functions in their work devoted to rigorous lower and upper bounds to the dynamic polarizability of two-electron atoms. Sims and Rumble [36] used this type of wave function in the variation-perturbation calculations of static polarizability of four-electron atoms. The Kołos-Wolniewicz wave function was applied to both static [37,38] and dynamic [39-44] dipole polarizability of $\mathrm{H}_{2}$ in the ground and excited states. In principle, $\Psi_{\lambda}^{(1)}$ should be optimized for each frequency separately. So far, however, only the wave functions with optimized linear parameters have been reported in literature. Exceptionally, simple adjustments of the nonlinear parameters with respect to the static polarizability have been performed [35]. Only very recently, Cencek et al. [6] fully optimized first- and second-order ECG wave functions of He but also with respect to the static properties. In the present paper, much more flexibility was added to both the method and the wave functions, as the nonlinear parameters of $\widetilde{\Psi}_{\lambda}^{(1)}$ were optimized with respect to the static and dynamic polarizabilities and also with respect to the lowest excitation energies. The optimization algorithm was similar to that applied to the unperturbed wave function [26], but the goal function was either $\alpha_{\lambda}(\omega)$ or the excited-state energy. The nonlinear optimization, although time consuming, was crucial for obtaining accurate results. More about our optimization scheme can be found in Refs. [20,24,26,45].

\section{Construction of the first-order wave function}

At the absorption frequency the dynamic polarizabilities exhibit discontinuities or poles. In practical calculations the poles appear at the frequencies $\omega_{l}=E_{l}-E_{0}(l=1,2, \ldots)$, where $E_{0}$ is the unperturbed state energy obtained from $\widetilde{\Psi}^{(0)}$ and is assumed to be known with very high accuracy. $E_{l}$ are consecutive eigenvalues obtained by diagonalization of the
Hamiltonian $H^{(0)}$ in the basis of the perturbation correction wave function $\widetilde{\Psi}_{\lambda}^{(1)}$ of the appropriate symmetry. As mentioned above, it is a common practice to optimize this basis set with respect to the static polarizability, not the energies $E_{l}$. As a consequence, the $\alpha_{\lambda}(0)$ values are recovered with reasonable accuracy but when the frequency departs from zero the accuracy of the $\alpha_{\lambda}(\omega)$ curve drops rapidly and, additionally, the positions of the poles appear too high on the frequency scale. Examples of such a tendency can be found even in the most accurate calculations employing explicitly correlated wave functions [39-44].

The procedure described below, based on the variationality of both the energy and the polarizability, allows this deficiency to be eliminated. It relies on the observation that if we merge a basis set of the length $K_{\lambda}^{(1)}$, whose nonlinear parameters were optimized with respect to the static polarizability, with a basis set of the length $K_{\lambda}^{l}$, optimized with respect to the energy of the $l$ th eigenvalue of an appropriate symmetry, then the resulting basis set of the size $K_{\lambda}^{\mathrm{F}}=K_{\lambda}^{(1)}$ $+K_{\lambda}^{l}$ gives $\alpha_{\lambda}\left(K_{\lambda}^{\mathrm{F}}\right) \geqslant \alpha_{\lambda}\left(K_{\lambda}^{(1)}\right)$ and simultaneously $E_{l}\left(K_{\lambda}^{\mathrm{F}}\right)$ $\leqslant E_{l}\left(K_{\lambda}^{l}\right)$, i.e., the combined basis set deteriorates neither the polarizability nor the excited state energy obtained from the separate basis sets. In this way we can generate a basis set that combines the advantages of its components and yields both accurate static polarizability and the position of the pole.

In general, the first-order correction wave function can be constructed by merging many basis sets, each optimized with different goal functions. In the present paper, the nonlinear parameters of the final expansion of $\widetilde{\Psi}_{\lambda}^{(1)}$ were generated in several separate optimization steps and the length $K_{\lambda}^{\mathrm{F}}$ of the final expansion was a sum of the sizes of the basis sets employed in these steps: $K_{\lambda}^{\mathrm{F}}=\sum_{j} K_{\lambda}^{(1)}\left(\omega_{j}\right)+\Sigma_{l} K_{\lambda}^{l}$. In the first group of steps, the nonlinear parameters were optimized variationally with respect to the polarizability by using the Hylleraas functional, Eq. (2.12), at selected frequencies $\omega_{j}$ $(j=0,1, \ldots)$. The size of the basis sets optimized in these steps was labeled $K_{\lambda}^{(1)}\left(\omega_{j}\right)$ with explicit dependence on the frequency in order to emphasize that the optimization was performed not only for the static polarizability but also at some frequencies from the range $0<\omega_{j}<E_{1}-E_{0}$ (in this range the Hylleraas variational principle is valid [31]). The second group of the steps generates the $K_{\lambda}^{l}$-term expansions with nonlinear variational parameters optimum with respect to the energy of the $l$ th root of the Hamiltonian diagonalized with the function of appropriate symmetry: $\lambda=1$ for $P$ states or $\lambda=2$ for states of $D$ symmetry. The final basis set of the size $K_{\lambda}^{\mathrm{F}}$ obtained in the above procedure was not optimized any further. Optimization of this basis would improve the selected goal quantity but deteriorate the rest of the features of the $\alpha_{\lambda}(\omega)$ function.

The final basis set constructed in this way has the following advantages over the basis generated in a single step. (i) It yields improved polarizabilities and excited-state energies without the time-consuming optimization of large basis sets. (ii) As the final basis set contains the basis functions of several excited states, it ensures that the subsequent poles of the 
TABLE I. Results of the test calculations.

\begin{tabular}{|c|c|c|c|}
\hline Property & Reference & Basis size & Value \\
\hline \multicolumn{4}{|c|}{$\mathrm{He}$} \\
\hline \multirow[t]{2}{*}{$E\left(1^{1} S\right)$} & Exact $[46]$ & & -2.9037243770341195983 \\
\hline & ECG & 600 & -2.903724377022 \\
\hline \multirow[t]{2}{*}{$\alpha_{1}(0)$} & Exact [5] & & $1.383192174455(1)$ \\
\hline & ECG & 1270 & 1.383192154 \\
\hline \multirow[t]{2}{*}{$\gamma_{1}(0)$} & Exact & & 1.0000000 \\
\hline & ECG & 1270 & 0.9999999 \\
\hline \multirow[t]{2}{*}{$E\left(2{ }^{1} P\right)$} & Exact [47] & & $-2.123843086498094(5)$ \\
\hline & ECG & 1270 & -2.1238430856 \\
\hline \multirow[t]{2}{*}{$\alpha_{2}(0)$} & Exact $[48]$ & & $2.445083101(2)$ \\
\hline & ECG & 1800 & 2.445083016 \\
\hline \multirow[t]{2}{*}{$\gamma_{2}(0)$} & Exact & & N/A \\
\hline & ECG & 1800 & 0.4076810 \\
\hline \multirow[t]{2}{*}{$E\left(3{ }^{1} D\right)$} & Exact [47] & & $-2.055620732852246(6)$ \\
\hline & ECG & 1800 & -2.05562073238 \\
\hline \multirow[t]{2}{*}{$E\left(4{ }^{1} D\right)$} & Exact $[47]$ & & $-2.031279846178687(7)$ \\
\hline & ECG & 1800 & -2.031279817 \\
\hline \multicolumn{4}{|c|}{$\mathrm{Li}$} \\
\hline \multirow[t]{2}{*}{$E\left(2^{2} S\right)$} & Exact [49] & & $-7.4780603236503(71)$ \\
\hline & ECG & 1536 & -7.4780603143 \\
\hline \multirow[t]{2}{*}{$\alpha_{1}(0)$} & Exact $[48]$ & & $164.111(2)$ \\
\hline & ECG & 3700 & 164.11171 \\
\hline \multirow[t]{2}{*}{$\gamma_{1}(0)$} & Exact & & 1.00000 \\
\hline & ECG & 3700 & 0.99973 \\
\hline \multirow[t]{2}{*}{$E\left(2{ }^{2} P\right)$} & Exact [49] & & $-7.410156531763(42)$ \\
\hline & ECG & 3700 & -7.41015622 \\
\hline \multirow[t]{2}{*}{$E\left(3^{2} P\right)$} & {$[51]$} & & -7.33714902 \\
\hline & ECG & 3700 & -7.3371490322 \\
\hline \multirow[t]{2}{*}{$E\left(4^{2} P\right)$} & {$[51]$} & & -7.31188330 \\
\hline & ECG & 3700 & -7.311864 \\
\hline \multirow[t]{2}{*}{$\alpha_{2}(0)$} & Exact $[48]$ & & $1423.266(5)$ \\
\hline & ECG & 2800 & 1423.282 \\
\hline \multirow[t]{2}{*}{$\gamma_{2}(0)$} & Exact & & N/A \\
\hline & ECG & 2800 & 0.7385 \\
\hline \multirow[t]{2}{*}{$E\left(3^{2} D\right)$} & Exact $[50]$ & & $-7.33552354110(43)$ \\
\hline & ECG & 2800 & -7.335519 \\
\hline \multirow[t]{2}{*}{$E\left(4^{2} D\right)$} & {$[51]$} & & -7.31118477 \\
\hline & ECG & 2800 & -7.31040 \\
\hline
\end{tabular}

$\alpha_{\lambda}(\omega)$ curves are extremely accurate-their positions correspond to the excitation energies of the states obtained from the $K_{\lambda}^{F}$-term expansions, i.e., are only a fraction of milliHartree in error. (iii) Forcing the correct position of the poles and optimization at $\omega>0$ ensures that the high accuracy of $\alpha_{\lambda}$ is preserved in a wide range of frequencies.

\section{RESULTS AND DISCUSSION}

\section{A. Test calculations}

The approach described in Sec. II C was tested on helium and lithium atoms, for which exact values of energies and polarizabilities are available from the literature. In Table I, appropriate variational energies and static polarizabilities ob-

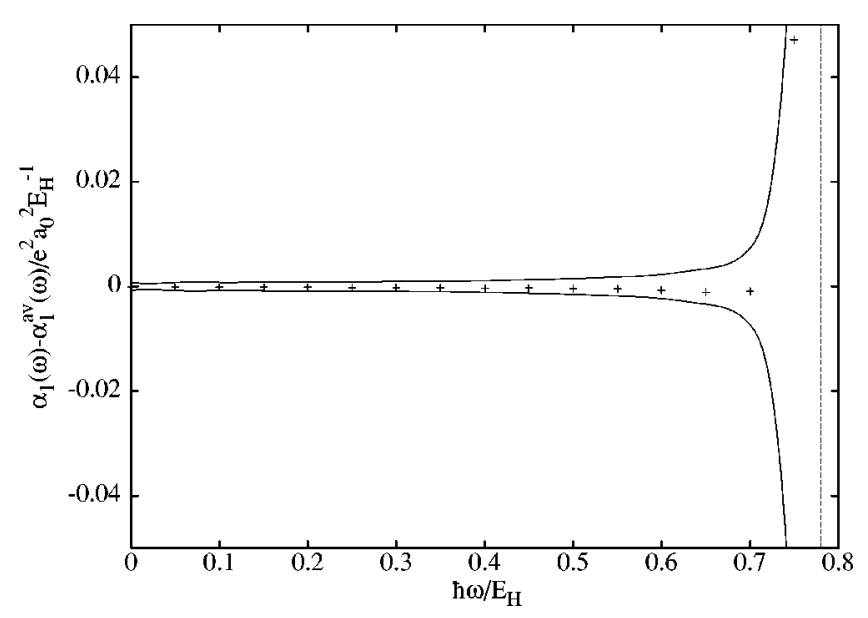

FIG. 1. Projection of the ECG dynamic dipole polarizability of $\mathrm{He}(+)$ on the area allowed by the Glover-Weinhold rigorous bounds (solid lines) [35] at frequencies up to the first excitation $\omega_{21 P}=0.779881291$.

tained using the variation-perturbation method from the ECG wave functions are confronted with the other most accurate energies available in the literature and with the polarizability values computed with the practically exact Hylleraas wave functions by using the sum over state procedure [5,46-51].

For the present calculations, the unperturbed ground-state wave function of He was taken from the work of Cencek and Kutzelnigg [17]. The 600-term ECG expansion gives the ground-state energy with $12 \times 10^{-12} E_{H}$ of error.

The dipole polarizability first-order expansion was assembled from 660-term $\alpha_{1}(0)$-optimized ECG wave function and 610-term ECG basis optimized with respect to $2{ }^{1} P$ state energy. The size of the final basis set was $K_{1}^{\mathrm{F}}=1270$ and $\alpha_{1}(0)=1.383192154$ obtained in this procedure differs from the exact value $[5,6]$ in ninth significant figure. The dipole shielding factor differs from unity by less than $10^{-7}$. The $2{ }^{1} P$ state energy computed in the final basis set is only 1 nanoHartree in error. The first pole of the dynamic polarizability curve is located (with the same error as the energy) at $\omega_{21 P}=0.779881291$.

The dynamic dipole polarizability of $\mathrm{He}$ was confronted with the rigorous upper and lower bounds given by Glover and Weinhold [35] for frequencies up to the second resonance. Though none of the components of $\widetilde{\Psi}_{1}^{(1)}$ was optimized at $\omega>0$, the $\alpha_{1}(\omega)$ curve fits perfectly those bounds. Figures 1 and 2 show two curves constructed from Glover and Weinhold's data: $\alpha_{1}^{\mathrm{ub}}(\omega)-\alpha_{1}^{\mathrm{av}}(\omega)$, and $\alpha_{1}^{\mathrm{lb}}(\omega)$ $-\alpha_{1}^{\mathrm{av}}(\omega)$, compared with the $\alpha_{1}(\omega)-\alpha_{1}^{\mathrm{av}}(\omega)$, curve obtained in this work. The "ub" and "lb" superscripts mean the rigorous upper and, respectively, lower bound curve, and "av" is an arithmetic average of them.

For the quadrupole polarizability, $\widetilde{\Psi}_{2}^{(1)}$ was built of three 600-term ECG basis sets: one set optimized with respect to $\alpha_{2}(0)$ and two sets with respect to $3{ }^{1} D$ and $4{ }^{1} D$ state energies. The final 1800-term expansion recovered $\alpha_{2}(0)$ $=2.445083016$ with a relative error of $3 \times 10^{-8}$. The poles of the $\alpha_{2}(\omega)$ function are located at $\omega_{3}{ }^{1} D=0.848103644$ and $\omega_{4}{ }^{1} D=0.8724445$ with all quoted figures being exact. 


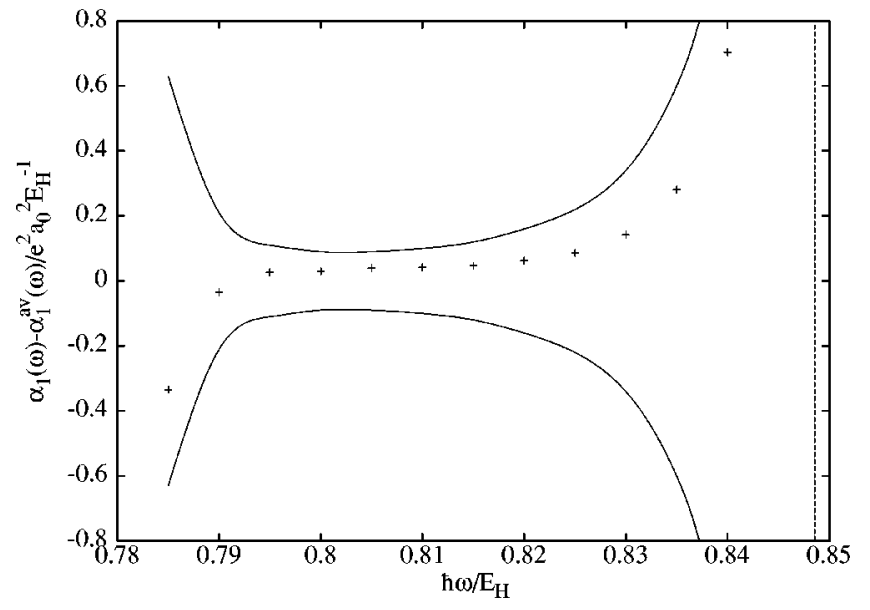

FIG. 2. As in Fig. 1 but at frequencies between the first and second excitation $\omega_{3}{ }^{1} P=0.8485961$.

The quadrupole shielding factor $\gamma_{2}(0)=0.4076810$ is the most accurate estimation of this quantity in literature. Previous estimations of $\gamma_{2}(0)$ come from late fifties: 0.424 [7], 0.416 [52], and 0.413 [53].

In the case of lithium atom, $\widetilde{\Psi}^{(0)}$ was chosen as 1536term ECG expansion of Cencek [54], which yields the ground-state energy with an error of $9.3 \times 10^{-9} E_{H}$.

Four basis sets of $P$ symmetry were combined to get the final 3700-term ECG expansion of $\widetilde{\Psi}_{1}^{(1)}$ : 1200-term basis optimized with respect to $\alpha_{1}(0)$, one 1300-term $2^{2} P$-optimized basis set, and two 600-term basis sets optimized with respect to the energy of the $3^{2} P$ and $4{ }^{2} P$ states. The final dipole polarizability $\alpha_{1}(0)=164.11171$ agrees perfectly with Yan et al. result 164.111(2) [48] (see Table I). The dipole shielding factor is equal to 0.99973 . The excitation energy to $2^{2} P$ state is $0.0679041 E_{H}$ with 3 $\times 10^{-7} E_{H}$ of error. For the next two excited ${ }^{2} P$ states no exact calculations are available in literature. The most accurate to date are those by Pestka and Woźnicki [51]. For $3{ }^{2} P$ state the present ECG calculations give the variational upper bound to the energy that is $0.01 \mu E_{H}$ lower than the energy cited in Ref. [51]. For the $4{ }^{2} P$ state their energy is by $20 \mu E_{H}$ lower that the ECG energy. The appropriate positions of the dipole polarizability poles are $\omega_{3}{ }^{2} P=0.1409113$ and $\omega_{4}{ }^{2} P=0.16620$.

For the calculation of the quadrupole properties a 1000term $\alpha_{2}(0)$-optimized basis was combined with 1200-term $3{ }^{2} \mathrm{D}$-optimized and 600 -term $4{ }^{2} \mathrm{D}$-optimized basis sets. The final 2800-term $\widetilde{\Psi}_{2}^{(1)}$ gave $\alpha_{2}(0)=1423.282$ compared to 1423.266(5) $e^{2} a_{0}^{4} E_{H}^{-1}$ obtained by Yan et al. [48]. For unknown reasons these two results differ by more or less three times their estimated error bar. The value of $\gamma_{2}(0)=0.7385$, which can be compared with 0.7156 estimated by Mahapatra and Rao [55].

Encouragingly the high accuracy of the test results supports the assertion that the above-described method of construction of $\widetilde{\Psi}_{\lambda}^{(1)}$ from the ECG functions has a potential capability of yielding accurate results also for larger systems including beryllium atom.

\section{B. Convergence of the static properties of beryllium}

In principle, the Hylleraas functional yields polarizabilities that are lower bounds to the exact values. However, the computed polarizabilities would represent rigorous lower bounds only if an exact $\Psi^{(0)}$ was used in solving the variation-perturbation equations. It is known that the Hylleraas functional (2.12) is very sensitive to the quality of the unperturbed wave function. The leading errors in $\alpha_{\lambda}$ are of the second order in the error of $\widetilde{\Psi}_{ \pm}^{(1)}$, but only of the first order in the error of $\widetilde{\Psi}^{(0)}[35,56]$. Therefore, particular effort was put on the construction of the wave function describing the unperturbed atom.

The unperturbed beryllium atom wave functions were generated variationally for many expansion lengths, $K^{(0)}$ $=50, \ldots, 1600$. The wave functions with $K^{(0)} \leqslant 1200$ were exactly those of Ref. [26]. The only new wave function with $K^{(0)}=1600$ yields the nonrelativistic energy of - $14.667355536 E_{H}$, which is the lowest variational energy of the ground-state beryllium to date. The estimated error of the energy is less than $1 \mathrm{~cm}^{-1}$. Weinhold presented the formula [57] for the rigorous lower bound to polarizability even when both wave functions are only approximate. This formula becomes equivalent to the Hylleraas result in the limit $S \rightarrow 1$, where $S=\left\langle\Psi^{(0)} \mid \Psi^{(0)}\right\rangle$. The value of $S$, a measure of quality of $\Psi^{(0)}$, can be estimated using the Eckart's [58] or the Weinberger's [59] inequality. For the 1600term ECG wave function, the first one yields $S$ $\geqslant 0.9999910$, the second-stronger criterion- $S$ $\geqslant 0.9999920$. Even the Weinberger's bound is known to give too weak an estimation [60], and the true overlap is still closer to unity. Very high accuracy of the $\Psi^{(0)}$ applied in the final calculations allows the error originating from the unperturbed wave function to be minimized and in practice the variationality of the functional (2.12) is preserved with good precision.

Table II illustrates the influence of the choice of $\widetilde{\Psi}^{(0)}$ on the static properties evaluated with well-optimized 1200term first-order wave function. For the smallest expansions the dipole polarizability decreases with the growing basis size. Only beginning with $K^{(0)}=100$ it converges monotonically to the final value yielding five stable digits. It is seen that $\alpha_{1}(0)$ obtained even with the smallest $K^{(0)}$ differs from that obtained with $K^{(0)}=1600$ by less than $0.25 \%$. The quadrupole polarizability behaves more regularly and grows monotonically in the whole range of $K^{(0)}$ displayed in Table II, yielding four converged figures. Although, for $\alpha_{2}(0)$ the convergence is slightly slower than in the dipole case, already $K^{(0)}=150$ yields $\alpha_{2}(0)$ within $1 \%$ of that obtained with $K^{(0)}=1600$.

The value of $\gamma_{1}(0)$, which for the neutral Be atom is known a priori to be equal 1, was evaluated using exactly the same zeroth- and first-order wave function as $\alpha_{1}$. Deviations of the computed $\gamma_{1}(0)$ from unity can be seen as a rough measure of quality of the pair of wave functions involved in the computations. From Table II, we see that beginning with $K^{(0)}=300, \gamma_{1}(0)$ grows monotonically towards 1. Less regular is the behavior of $\gamma_{2}(0)$ which 
TABLE II. Influence of the quality of the zeroth-order wave function on the static polarizabilities and shielding factors. $K_{\lambda}^{(1)}=1200$ and $E^{(0)}$ of Eqs. (2.11) from the second column.

\begin{tabular}{lccccc}
\hline \hline$K^{(0)}$ & $E^{(0)} / E_{H}$ & $\alpha_{1}(0)$ & $\gamma_{1}(0)$ & $\alpha_{2}(0)$ & $\gamma_{2}(0)$ \\
\hline 50 & -14.665053934 & 37.6897 & 1.01489 & 289.192 & 1.5402 \\
75 & -14.666444767 & 37.6771 & 1.00131 & 292.822 & 1.5215 \\
100 & -14.666892196 & 37.6675 & 0.99827 & 295.517 & 1.5145 \\
150 & -14.667185772 & 37.7023 & 0.99550 & 298.084 & 1.5121 \\
200 & -14.667271965 & 37.7304 & 0.99732 & 299.480 & 1.5136 \\
300 & -14.667315415 & 37.7328 & 0.99714 & 299.855 & 1.4868 \\
400 & -14.667335167 & 37.7382 & 0.99730 & 300.205 & 1.5011 \\
600 & -14.667350195 & 37.7508 & 0.99806 & 300.771 & 1.4903 \\
800 & -14.667353781 & 37.7530 & 0.99873 & 300.888 & 1.4871 \\
1200 & -14.667355022 & 37.7535 & 0.99911 & 300.923 & 1.4883 \\
1600 & -14.667355536 & 37.7536 & 0.99911 & 300.932 & 1.4875 \\
\hline \hline
\end{tabular}

oscillates around the final value in order to stabilize four significant figures.

A similar analysis of the convergence was performed for $\widetilde{\Psi}_{\lambda}^{(1)}$. Table III contains the polarizabilities and shielding factors computed with a series of the first-order wave functions with successively doubled expansions. In their optimization, as the $\widetilde{\Psi}^{(0)}$, the 1600 -term ECG wave function was employed. The first-order wave functions of increasing expansion size $K_{\lambda}^{(1)}$ were optimized with respect to $\alpha_{\lambda}(0)$ independently of each other. The static properties converge with the $\widetilde{\Psi}_{\lambda}^{(1)}$ expansion length slower than it was observed for $\Psi^{(0)}$. The last doubling of the $K_{\lambda}^{(1)}$ changes $\alpha_{1}(0)$ by $0.02 \%$ and $\alpha_{2}(0)$ by $0.06 \%$. In contrast to $\widetilde{\Psi}^{(0)}$ the smallest $\widetilde{\Psi}_{\lambda}^{(1)}$ expansions give results far from the final ones.

In the convergence analysis presented above, the zerothand first-order basis sets were of similar sizes. The apparently slower convergence of $\alpha_{\lambda}$ with $K_{\lambda}^{(1)}$ than with $K^{(0)}$ indicates that to saturate the functional space of $\Psi_{\lambda}^{(1)}$ still longer expansions have to be used. We emphasize here that $\widetilde{\Psi}_{\lambda}^{(1)}$ were not optimized with respect to $\gamma_{\lambda}$. Both shielding factors were obtained with the $\widetilde{\Psi}_{\lambda}^{(1)}$ optimized with respect to $\alpha_{\lambda}$ alone and this justifies some irregularities in the $\gamma_{\lambda}$ convergence. Nevertheless, a relative accuracy better than $0.1 \%$ was achieved for both $\gamma_{1}$ and $\gamma_{2}$. The 1600-term ECG wave function employed to approximate $\Psi^{(0)}$, though very accurate, is not strictly exact. Therefore, the polarizability results presented here are not rigorously variational. The pos-

TABLE III. Convergence of the static properties with the expansion size $\left(K_{\lambda}^{(1)}\right)$ of the first-order wave function. $K^{(0)}=1600$ and $E^{(0)}=-14.667355536 E_{H}$.

\begin{tabular}{lcccc}
\hline \hline$K_{\lambda}^{(1)}$ & $\alpha_{1}(0)$ & $\gamma_{1}(0)$ & $\alpha_{2}(0)$ & $\gamma_{2}(0)$ \\
\hline 75 & 37.0952 & 0.88401 & 283.173 & 1.8015 \\
150 & 37.5774 & 0.96364 & 294.890 & 1.3384 \\
300 & 37.7154 & 0.98844 & 299.691 & 1.2607 \\
600 & 37.7462 & 0.99688 & 300.760 & 1.4184 \\
1200 & 37.7536 & 0.99911 & 300.932 & 1.4875 \\
\hline \hline
\end{tabular}

sible errors, however, manifest themselves only at further significant figures, which was illustrated by the test results presented in Sec. III A and the above convergence analysis.

\section{Construction of the final first-order wave functions of beryllium}

In all the individual $\alpha_{\lambda}$ optimizations and in the final calculations reported in this section, the best available 1600-term wave function was employed as $\Psi^{(0)}$ and the corresponding unperturbed-state energy $E^{(0)}=$ $-14.667355536 E_{H}$ was assumed.

The final first-order wave function of $P$ symmetry with $K_{1}^{\mathrm{F}}=4800$ was constructed according to the prescription given in Sec. II C. It was assembled from two polarizabilityoptimized and three energy-optimized basis sets of the following sizes: $K_{1}^{(1)}(0)=1200\left[\alpha_{1}(0)=37.7536\right]$, $K_{1}^{(1)}(0.19)=1200\left[\alpha_{1}(0.19)=912.976\right], K_{1}^{2}{ }^{1} P=1200, K_{1}^{3{ }^{1} P}$ $=600, K_{1}^{4}{ }^{1} P=600$. The partial and the final results of the $P$-state energy spectrum are compared directly in Table IV. In the square brackets, the polarizabilities obtained in the particular optimizations are quoted for comparison with pertinent final values listed in Table VI. This comparison gives an idea on the gain obtained when moving from the $E_{l}$-optimized wave function to the final wave function. For example, the energy of the $2^{2} P$ state decreased by $2.3 \times 10^{-6} E_{H}$ when computed with the final wave function compared to the original 1200-term energy-optimized wave function [27]. This improvement is much larger for the higher, less carefully optimized states (see Table IV).

The basis set for $\tilde{\Psi}_{2}^{(1)}\left(K_{2}^{\mathrm{F}}=4400\right)$ was assembled from the following partial basis sets: 1200-term $\alpha_{2}(0)$-optimized $\left[\alpha_{2}(0)=300.932\right], 600$-term $\alpha_{2}(0.25)$-optimized $\left[\alpha_{2}(0.25)\right.$ $=2857.01]$, 1200-term $E^{3}{ }^{1} D_{\text {-optimized, 600-term } E^{4}{ }^{1} D_{-}}$ optimized, and 800-term $E^{5}{ }^{1} D$-optimized. The excited-state energies resulting from these and the final basis sets are listed in Table IV.

In the case of the dynamic polarizability, the gain from the methodology applied here is well illustrated by the following example. The dipole polarizability at $\omega=0.19$ obtained from 
TABLE IV. ${ }^{1} P$ and ${ }^{1} D$ state energies of Be from the ECG wave function. Excitation energies computed with respect to the groundstate energy $-14.667360 E_{H}$ [26]. The atom-based Rydberg constant $R=109730.64 \mathrm{~cm}^{-1}$ was used to convert the excitation energies from $E_{H}$ to $\mathrm{cm}^{-1}$.

\begin{tabular}{|c|c|c|c|}
\hline \multirow[b]{2}{*}{ Basis size } & \multirow[b]{2}{*}{ Energy/ $E_{H}$} & \multicolumn{2}{|c|}{ Excitation energy } \\
\hline & & $E_{H}$ & $\mathrm{~cm}^{-1}$ \\
\hline \multicolumn{4}{|c|}{$2 s 2 p 2{ }^{1} P$} \\
\hline$K_{1}^{(1)}=1200$ & -14.47344202 & 0.193918 & 42557 \\
\hline$K_{1}^{\mathrm{F}}=4800$ & -14.47344433 & 0.193916 & $42557(42565.35)^{\mathrm{a}}$ \\
\hline \multicolumn{4}{|c|}{$2 s 3 p 3{ }^{1} P$} \\
\hline$K_{1}^{(1)}=600$ & -14.39304978 & 0.274310 & 60200 \\
\hline$K_{1}^{\mathrm{F}}=4800$ & -14.39311393 & 0.274246 & $60186(60187.34)^{\mathrm{a}}$ \\
\hline \multicolumn{4}{|c|}{$2 s 4 p 4{ }^{1} P$} \\
\hline$K_{1}^{(1)}=600$ & -14.36176167 & 0.305598 & 67067 \\
\hline$K_{1}^{\mathrm{F}}=4800$ & -14.36178921 & 0.305571 & $67061(67034.70)^{\mathrm{a}}$ \\
\hline \multicolumn{4}{|c|}{$2 p^{2} 3{ }^{1} D$} \\
\hline$K_{2}^{(1)}=1200$ & -14.4081811 & 0.25917 & 56879 \\
\hline$K_{2}^{\mathrm{F}}=4400$ & -14.4081923 & 0.25916 & $56876(56882.43)^{\mathrm{a}}$ \\
\hline \multicolumn{4}{|c|}{$2 s 3 d 4{ }^{1} D$} \\
\hline$K_{2}^{(1)}=600$ & -14.3733384 & 0.29402 & 64525 \\
\hline$K_{2}^{\mathrm{F}}=4400$ & -14.3735631 & 0.29379 & $64476(64428.31)^{\mathrm{a}}$ \\
\hline \multicolumn{4}{|c|}{$2 s 4 d 5{ }^{1} D$} \\
\hline$K_{2}^{(1)}=800$ & -14.3488478 & 0.31851 & 69901 \\
\hline$K_{2}^{\mathrm{F}}=4400$ & -14.3492532 & 0.31811 & $69812(68780.86)^{\mathrm{a}}$ \\
\hline
\end{tabular}

${ }^{a}$ Experimental data from Ref. [61].

the $\alpha_{1}(0)$-optimized wave function is ca. $906 e^{2} a_{0}^{2} E_{H}^{-1}$ and from the $\alpha_{1}(0.19)$-optimized wave function of the same size it is by $7 e^{2} a_{0}^{2} E_{H}^{-1}$ larger, whereas the final first-order wave function improves further this value by four units. The gain observed in the quadrupole polarizability is even more pronounced: $\alpha_{2}(0.25)$ increases from $2667 e^{2} a_{0}^{4} E_{H}^{-1}$ by 190 units when moving from the $\alpha_{2}(0)$ - to $\alpha_{2}(0.25)$-optimized function, and by another $64 e^{2} a_{0}^{4} E_{H}^{-1}$ in the final calculations.

At this point some comment on the nonstatic polarizability optimization is relevant. The values of $\alpha_{\lambda}(\omega)$ are arithmetic sums of the plus and minus components (Sec. II B) computed from two separate first-order corrections, $\widetilde{\Psi}_{\lambda \pm}^{(1)}$. For a fixed $\lambda, \widetilde{\Psi}_{\lambda+}^{(1)}$ and $\widetilde{\Psi}_{\lambda-}^{(1)}$ are expanded in the same basis but, because they are solutions to two different equations, Eqs. (2.11), they differ in the linear expansion coefficients. The $\alpha_{\lambda+}(\omega)$ functions vary slowly with $\omega$ and exhibit no poles. The $\alpha_{\lambda-}$ components are responsible for large variations in the polarizability value and for the presence of the poles. The nonstatic optimization was performed with respect to the latter component at a frequency slightly smaller than the first resonance energy. This ensures correct values of the dynamic polarizability curve at frequencies far from zero.

As a by-product of this work, upper bounds to the energies of several excited $P$ and $D$ states of beryllium were obtained. These are the lowest variational energies known to date. Table IV collects the energies of three lowest states of both symmetries calculated with the final basis sets. For the sake of comparison, the energies obtained in the particular
TABLE V. Comparison of the static polarizabilities with the selected literature data computed at the correlated level.

\begin{tabular}{lcl}
\hline \hline$\alpha_{1}$ & Method & \multicolumn{1}{c}{ Reference } \\
\hline $37.0 \pm 3.4$ & CI & Kolker and Michels [34] \\
$36.5 \pm 1.7$ & MCSCF & Stevens and Billingsley, [68] \\
$36.6 \pm 0.8$ & VP & Sims and Rumble [36] \\
37.84 & PNO-CEPA & Werner and Meyer [69] \\
37.59 & VP CI & Figari et al. [66] \\
37.69 & CI & Müller et al. [70] \\
37.64 & MCTDHF & Graham and Yeager [71] \\
$37.3 \pm 0.7$ & MPPT(4) & Maroulis and Thakkar [63] \\
37.70 & CCSDT-1a & Urban et al. [72] \\
37.53 & FCI & Koch and Harrison [73] \\
37.62 & MCHF & Themelis and Nicolaides [74] \\
$37.73 \pm 0.05$ & CC-R12 & Tunega, Noga and Klopper [65] \\
37.6 & TDGI & Bégué, Merawa and Pouchan [62] \\
37.67 & CCSD(T) & Papadopoulos and Sadlej [75] \\
37.9 & QCISD(T) & Leung and Breckenridge [76] \\
37.755 & ECG & This work \\
\hline$\alpha 32$ & & \\
\hline $301.8+3.5$ & CEPA & Reinsch and Meyer [77] \\
299.4 & VP CI & Figari, Musso and Magnasco [66] \\
$298.8 \pm 2.6$ & MPPT(4) & Maroulis and Thakkar [63] \\
313.4 & MPPT(2) & Thakkar [67] \\
301.8 & MPPT(3) & Thakkar [67] \\
298.8 & CCD & Thakkar [67] \\
285.6 & TDGI & Bégué, Merawa and Pouchan [62] \\
300.96 & ECG & This work \\
\hline \hline & & \\
\hline & &
\end{tabular}

state optimization are also given. They illustrate the energy gain resulting from the methodology employed here. The accuracy of these energies can be estimated by a comparison of the excitation energies presented also in this table with the experimental lines available from NIST Atomic Spectra Database [61]. Note, however, that the theoretical values do not include relativistic or mass polarization effects.

\section{Comparison of the static properties with literature data}

A multitude of calculations of the static dipole polarizability of Be have been reported. Many of them were performed using methods incorporating electron correlation. An extensive listing of such results could be created from tables in Refs. [36,62-64]. Most of the numerical values fits in the (36;39) interval. On the basis of the present ECG lower bound equal to $37.755 e^{2} a_{0}^{2} E_{H}^{-1}$, a large part of them could be ruled out. Table $\mathrm{V}$ contains only selected results, obtained by correlated methods, which do not differ much from the present prediction, even when they are not above the ECG lower bound.

Some papers deserve separate comments. Sims and Rumble [36] applied the Weinhold's rigorous lower-bound procedure to beryllium atom. According to the present standards, however, their wave functions were of low quality (their $\widetilde{\Psi}^{(0)}$ and $\widetilde{\Psi}_{1}^{(1)}$ gave $E^{2}{ }^{1} S$ and $E^{2}{ }^{1} P$ energies with er- 
TABLE VI. Dynamic dipole and quadrupole polarizabilities and shielding factors of beryllium atom at selected frequencies $\omega$. $K^{(0)}=1600, \quad K_{1}^{\mathrm{F}}=4800, \quad K_{2}^{\mathrm{F}}=4400, \quad$ and $\quad E^{(0)}=$ $-14.667355536 E_{H}$.

\begin{tabular}{|c|c|c|c|c|c|}
\hline$\omega$ & $\begin{array}{c}\alpha_{1}(\omega) / \\
e^{2} a_{0}^{2} E_{H}^{-1}\end{array}$ & $\gamma_{1}(\omega)$ & $\omega$ & $\begin{array}{c}\alpha_{2}(\omega) / \\
e^{2} a_{0}^{4} E_{H}^{-1}\end{array}$ & $\gamma_{2}(\omega)$ \\
\hline 0.00 & 37.754950 & 0.998434 & 0.00 & 300.9589 & 1.4769 \\
\hline 0.01 & 37.852988 & 0.999382 & 0.01 & 301.3196 & 1.4790 \\
\hline 0.02 & 38.150249 & 1.002253 & 0.02 & 302.4072 & 1.4855 \\
\hline 0.03 & 38.656440 & 1.007140 & 0.03 & 304.2396 & 1.4963 \\
\hline 0.04 & 39.388631 & 1.014205 & 0.04 & 306.8470 & 1.5118 \\
\hline 0.05 & 40.372756 & 1.023691 & 0.05 & 310.2735 & 1.5322 \\
\hline 0.06 & 41.646046 & 1.035951 & 0.06 & 314.5793 & 1.5580 \\
\hline 0.07 & 43.260859 & 1.051479 & 0.07 & 319.8436 & 1.5897 \\
\hline 0.08 & 45.290698 & 1.070968 & 0.08 & 326.1685 & 1.6281 \\
\hline 0.09 & 47.839844 & 1.095402 & 0.09 & 333.6848 & 1.6741 \\
\hline 0.10 & 51.059268 & 1.126204 & 0.10 & 342.5598 & 1.7290 \\
\hline 0.11 & 55.174019 & 1.165495 & 0.11 & 353.0089 & 1.7942 \\
\hline 0.12 & 60.532939 & 1.216561 & 0.12 & 365.3113 & 1.8720 \\
\hline 0.13 & 67.705127 & 1.284761 & 0.13 & 379.8335 & 1.9651 \\
\hline 0.14 & 77.683821 & 1.379449 & 0.14 & 397.0648 & 2.0773 \\
\hline 0.15 & 92.368950 & 1.518506 & 0.15 & 417.6713 & 2.2138 \\
\hline 0.16 & 115.90351 & 1.740924 & 0.16 & 442.5833 & 2.3819 \\
\hline 0.17 & 159.36329 & 2.150930 & 0.17 & 473.1395 & 2.5925 \\
\hline 0.18 & 265.67376 & 3.152431 & 0.18 & 511.3405 & 2.8619 \\
\hline \multirow[t]{2}{*}{0.19} & 916.76453 & 9.280019 & 0.19 & 560.3198 & 3.2162 \\
\hline & & & 0.20 & 625.2886 & 3.6995 \\
\hline 0.20 & -571.44201 & -4.721303 & 0.21 & 715.6259 & 4.3923 \\
\hline 0.21 & -209.86046 & -1.317393 & 0.22 & 850.1554 & 5.4586 \\
\hline 0.22 & -125.56646 & -0.522272 & 0.23 & 1073.241 & 7.2900 \\
\hline 0.23 & -88.024602 & -0.166683 & 0.24 & 1521.165 & 11.103 \\
\hline 0.24 & -66.761519 & 0.036312 & 0.25 & 2922.734 & 23.459 \\
\hline 0.25 & -52.992792 & 0.169870 & & & \\
\hline 0.26 & -43.086922 & 0.269998 & 0.27 & -1916.888 & -21.399 \\
\hline \multirow[t]{2}{*}{0.27} & -33.515341 & 0.388546 & 0.28 & -781.6915 & -12.502 \\
\hline & & & 0.29 & -171.7518 & -13.531 \\
\hline 0.28 & -34.633425 & 0.317771 & & & \\
\hline 0.29 & -28.630317 & 0.395380 & 0.30 & -306.1427 & -3.1167 \\
\hline 0.30 & -24.689391 & 0.441820 & 0.31 & 141.5432 & -5.8349 \\
\hline
\end{tabular}

rors of $810 \times 10^{-6}$ and $980 \times 10^{-6} E_{H}$, respectively), and the lower bound $35.75 e^{2} a_{0}^{2} E_{H}^{-1}$ was too low to be of discriminating quality. Sims and Rumble obtained also $\alpha_{1}(0)$ from the variation-perturbation calculation. But the error bars assigned to their result $36.6 \pm 0.8$ were too narrow to reach the ECG lower bound.

To date the most accurate prediction of the static polarizability $\alpha_{1}(0)=37.73 \pm 0.05$ comes from the calculations by Tunega et al. [65] who used the finite-field technique in combination with the explicitly correlated version of the coupledcluster method (CC-R12) and a high-quality basis set. The uncertainty, although narrow, is wide enough to overlap with the region allowed by the ECG lower bound.

The correlated calculations of the quadrupole static polarizability are less common in literature. Table $\mathrm{V}$ shows that the predicted $\alpha_{2}(0)$ cover a wide range of numerical values,

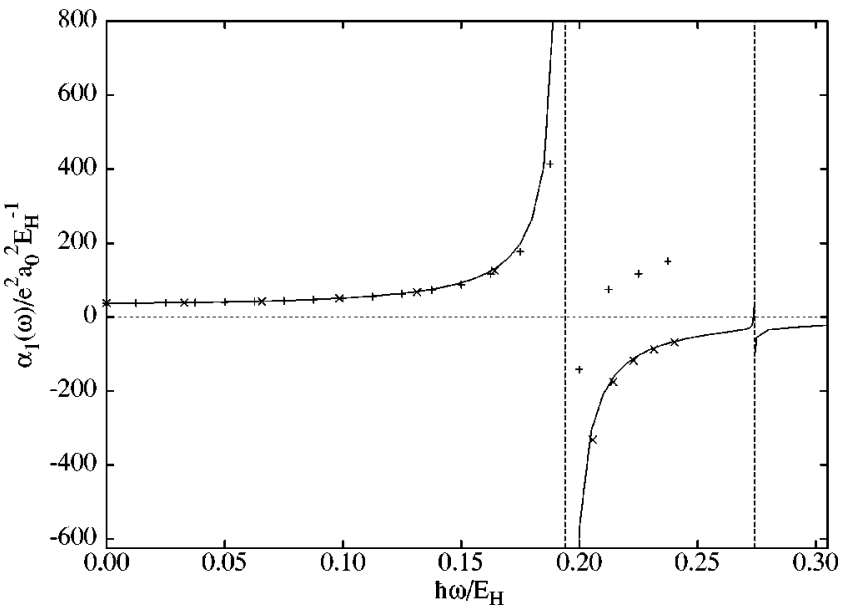

FIG. 3. Dynamic dipole polarizability of Be from ECG calculations (solid line). The two poles at $\omega=0.193916$ and $\omega$ $=0.274246$, result from the $2{ }^{1} P \leftarrow 2{ }^{1} S$ and $3{ }^{1} P \leftarrow 2{ }^{1} S$ transitions, respectively. For comparison, the time-dependent gauge invariant results by Bégué et al. [62] (+) and full configuration interaction results by Koch and Harrison [73] $(\times)$ are also presented.

$285.5 \leqslant \alpha_{2}(0) \leqslant 313.4$. The ECG value more or less halves this interval so that some of these results $[62,66,67]$ fall below the ECG lower bound $\alpha_{2}(0)=300.96$.

There are no accurate calculations of shielding factors in literature. The present calculations yield $\gamma_{1}(0)=0.9984$ with an error of about $16 \times 10^{-4}$. This relatively large error is justified by the fact that the wave functions employed in evaluation of $\gamma_{1}$ have not been optimized with respect to this quantity and that the electric field operator emphasizes the space near the nucleus whereas the dipole moment operator used in the optimization of $\widetilde{\Psi}_{1}^{(1)}$ - the space remote from the nucleus.

The static quadrupole shielding factor has been recently obtained from the quadrupole moment data of helium dimer at very short internuclear distances [29]. The present $\gamma_{2}(0)$ $=1.4769$ confirms the estimated $\gamma_{2}(0)=1.5(1)$ of Ref. [29]. There are also other, 40-year old, results for $\gamma_{2}(0): 1.24$ and 0.77 by Dalgarno and McNamee [78], 0.72 by Cohen et al. [79], and 0.75 by Kelly [80].

\section{E. Final dynamic polarizabilities and shielding factors}

The recommended dynamic polarizability and shielding factor curves at frequencies up to the third resonances $(\omega$ $\leqslant 0.31$ ) are collected in Table VI and illustrated in Figs. 3 and 4 . The dipole shielding factor curve mimics the shape of $\alpha_{\lambda}(\omega)$ and there is no need to draw $\gamma_{1}(\omega)$ separately. A rough similarity of $\alpha_{1}(\omega)$ and $\gamma_{1}(\omega)$ curves can be understood in view of the following relation $[81,82]$ :

$$
\gamma_{1}(\omega)=\frac{\omega^{2}}{Z} \alpha_{1}(\omega)+\gamma_{1}(0)
$$

The position of the first pole of $\alpha_{1}(\omega)\left(\omega_{2}{ }^{1}{ }_{P}=0.193916\right)$, corresponding to $2{ }^{1} P \leftarrow 2{ }^{1} S$ transition, is merely about $0.02 \times 10^{-3} E_{H}$ in error [27]. This fact in connection with the 


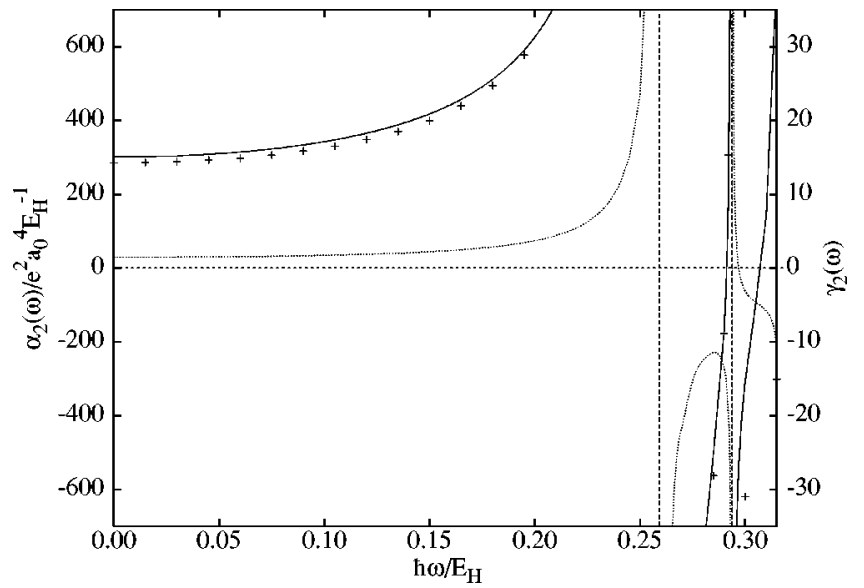

FIG. 4. Dynamic quadrupole polarizability (solid line, left axis) and quadrupole shielding factor (dotted line, right axis) of Be from ECG calculations. $3{ }^{1} D \leftarrow 2{ }^{1} S$ and $4{ }^{1} D \leftarrow 2{ }^{1} S$ transitions are marked at $\omega=0.25916$ and $\omega_{4}{ }^{1} D=0.29375$, respectively. Additionally, results of TDGI calculations of $\alpha_{2}$ by Bégué et al. [62] are shown as + .

dynamic-polarizability-optimized component of $\widetilde{\Psi}_{1}^{(1)}$ make the first branch of the $\alpha_{1}(\omega)$ curve very accurate-it is presumed that at least four significant figures remain correct in the whole interval excluding the nearest neighborhood of the pole. The location of the two remaining poles is less precise, but still their errors are much bellow $1 \times 10^{-3} E_{H}$. An estimation of their accuracy can be based on the comparison with the experimental lines collected in Table IV. The first pole of $\alpha_{2}(\omega)$, corresponding to $3{ }^{1} D \leftarrow 2{ }^{1} S$ resonance, appears at $\omega_{3}{ }^{1} D=0.25916$. The second, at $\omega_{4}{ }^{1} D$ $=0.29375$, results from $4{ }^{1} D \leftarrow 2{ }^{1} S$ transition. The overall shape of the $\alpha_{2}(\omega)$ curve is similar to $\alpha_{1}(\omega)$, i.e., in the intervals between poles it is a monotonically growing function of frequency (Fig. 4). The second and higher branches of $\alpha_{\lambda}(\omega)$ go from $-\infty$ to $+\infty$. They must, therefore, at some frequencies cross the $\alpha_{\lambda}=0$ line. Of some interest might be the location of the lowest frequency at which $\alpha_{\lambda}$ vanish $\left(\omega_{0}\right)$. Unfortunately, for both polarizabilities $\omega_{0}$ lies very close to the second resonance pole, so their values are uncertain. For the dipole polarizability $\omega_{0} \simeq 0.2737$, whereas the second pole lies at $\omega_{31}{ }^{1}=0.27425$. In the quadrupole case, $\omega_{0} \simeq 0.2912$ and the second pole appears at $\omega_{4}{ }^{1} D$ $=0.29375$. Contrary to the dipole case, the quadrupole shielding factor curve differs in shape from that of polarizability. This difference is particularly well visible in the second and third branches (see Fig. 4), e.g., there is a local maximum in $\gamma_{2}(\omega)$ at $\omega \approx 0.285$.

There are two calculations on the dynamic polarizabilities of $\mathrm{Be}$ reported in the literature. For the sake of comparison their results are displayed in Figs. 3 and 4.

Koch and Harrison [73] calculated the frequencydependent dipole polarizability at the full configuration interaction (FCI) level. Up to the first excitation frequency their curve is below the present ECG lower bound. At zero frequency, the difference between the ECG and their values is $0.32 e^{2} a_{0}^{2} E_{H}^{-1}$ and it increases with frequency to several units near $\omega=0.165$. Also at frequencies above the first resonance the FCI curve goes several percent below the ECG curve. The first resonance energy quoted in [73] is ca. 1.4 $\times 10^{-3} E_{H}$ above the correct value.

Recently Bégué et al. [62] have calculated the dynamic dipole and quadrupole polarizabilities of the ground and excited ${ }^{1} S$ states of beryllium by using time-dependent gauge invariant method (TDGI). The poles of the TDGI $\alpha_{1}(\omega)$ appear at positions higher than those of the resonance frequencies by approximately $2 \times 10^{-3} E_{H}$ and $11 \times 10^{-3} E_{H}$ for the first and the second resonance, respectively. Up to the first resonance, their $\alpha_{1}(\omega)$ curve goes below the ECG lower bound with a discrepancy increasing with frequency. In the second interval of frequencies, the TDGI polarizability lies significantly higher than the present one and those calculated by Koch and Harrison (see Fig. 4). There is no rigorous proof that the Hylleraas functional gives the lower bound to the exact polarizability also at frequencies above the first resonance, however, the convergence of both dipole and quadrupole polarizabilities with increasing quality of the wave functions suggests that this is the case. Anyhow, the discrepancy between Bégué $e t$ al. and ECG results is so large that their calculations cannot be recognized as more accurate but rather contaminated by some systematic error. No such discrepancy is observed for $\alpha_{2}(\omega)$.

The dynamic shielding factors data are very scarce in literature. For beryllium the only $\gamma_{1}(\omega)$ curve was calculated at the coupled Hartree-Fock level in 1969 by Kaveeshwar et al. [81]. This curve, though starts with the correct static value, grows too fast and has the first pole at $\omega=0.175$ (compared with the correct value 0.1939).

\section{F. Dispersion properties of Be}

For small frequencies the dynamic polarizabilities can be represented by a power series in $\omega^{2}$, the so-called Cauchy expansion,

$$
\alpha_{\lambda}(\omega)=\sum_{k=0}^{\infty} S_{\lambda}(-2 k-2) \omega^{2 k}
$$

with the coefficients $S_{\lambda}$ identified as the dipole or quadrupole oscillator sum rules. A polynomial fit to the data in Table VI in the range $0.0 \leqslant \omega \leqslant 0.10$ revealed the following values of the first few Cauchy moments (with the statistical uncertainty in the last digit): $S_{1}(-2)=37.755(37.6)$, $S_{1}(-4)=983.3(945.9), \quad S_{1}(-6)=2.26 \times 10^{4}\left(1.61 \times 10^{4}\right)$, $S_{1}(-8)=1.21 \times 10^{6}\left(1.54 \times 10^{6}\right)$. For the sake of comparison, values of $S_{1}$ obtained by Bégué et al. [62] are shown in parentheses. Similarly, $\alpha_{2}(\omega)$ expanded according to Eq. (3.2) yields the following quadrupole oscillator sums: $S_{2}(-2)=300.96, \quad S_{2}(-4)=3603.8, \quad S_{2}(-6)=4.66 \times 10^{4}$, $S_{2}(-8)=9.03 \times 10^{6}$.

The knowledge of the frequency dependence of the dipole polarizability enables an evaluation of other dispersion properties of hypothetical atomic vapors, like the refractive index or the Verdet constant [10]. The index of refraction, $n(\omega)$, is linked with $\alpha_{1}$ by the Lorentz-Lorenz equation 


$$
\frac{n^{2}-1}{n^{2}+2}=\frac{4 \pi N_{L}}{3} \alpha_{1}(\omega),
$$

where the Loschmidt's number $N_{L}=2.6867775(47)$ $\times 10^{25} \mathrm{~m}^{-3}$. Typically $n \approx 1$, and the left-hand side of Eq. (3.3) can be well approximated by $\frac{2}{3}(n-1)$ which, in connection with Eq. (3.2), leads to the following frequency dependence of $n$ :

$$
n-1=9.44 \times 10^{-4}+0.0246 \omega^{2}+0.565 \omega^{4}+30.3 \omega^{6} .
$$

Inserting the first few terms of Eq. (3.2) into the formula for the Verdet constant (expressed in $\operatorname{rad} e a_{0} \hbar^{-1}$ ) [83,84],

$$
V(\omega)=K \omega \frac{d \alpha_{1}(\omega)}{d \omega},
$$

where $K=0.91274213 \times 10^{-7}$ yields

$$
V(\omega)=1.795 \times 10^{-4} \omega^{2}+8.251 \times 10^{-3} \omega^{4}+0.6627 \omega^{6} .
$$

Jørgensen et al. [85] computed $V(\omega)$ for Be using the first (TDHF) and second (SOPPA) order polarization propagator methods. At small frequencies, the present $V(\omega)$ lies between the TDHF and SOPPA curves.

The numerical values considered above are valid at standard temperature and pressure at which beryllium is a solid metal. The temperature dependence of $n(\omega)$ and $V(\omega)$ is not considered here and it will be therefore difficult to verify experimentally the above results, but they may be of interest as a reference in theoretical study.

\section{SUMMARY}

The main advantage of the applied method is its variationality. The lower-bound polarizabilities obtained with the ECG wave functions allow the quality of the results obtained with other methods to be assessed. A comparison of the literature dipole and quadrupole polarizabilities with the lower bounds presented in this paper reveals that the majority of the literature data are underestimated.

The high quality of the unperturbed wave function in connection with the method of the construction of the first-order wave function ensures high accuracy of the polarizabilities and shielding factors in a wide range of frequencies and prediction of the correct positions of the poles. The results of these large-scale calculations can be recommended as benchmarks.

As a by-product of the procedure of construction of $\widetilde{\Psi}_{\lambda}^{(1)}$, new upper-bound energies of several lowest-excited states of Be and $3{ }^{2} P$ state of Li were obtained. Also, $\gamma_{2}(0)$ values of $\mathrm{He}$ and $\mathrm{Li}$ are the most accurate to date.

\section{ACKNOWLEDGMENTS}

I am indebted to Professor J. Rychlewski and Dr. W. Cencek for stimulating discussions and comments. This study was supported by the Polish State Committee for Scientific Research Grant Nos. 3 T09A 08716 and SPUB/COST/D9 and by a computing grant from Poznań Supercomputing and Networking Center.
[1] M.R. Moldover, J. Res. Natl. Inst. Stand. Technol. 103, 167 (1998).

[2] J.J. Hurly and M.R. Moldover, J. Res. Natl. Inst. Stand. Technol. 105, 667 (2000).

[3] L.R. Pendrill, J. Phys. B 29, 3581 (1996).

[4] P.J. Mohr and B.T. Taylor, Rev. Mod. Phys. 72, 351 (2000).

[5] K. Pachucki and J. Sapirstein, Phys. Rev. A 63, 12504 (2001).

[6] W. Cencek, K. Szalewicz, and B. Jeziorski, Phys. Rev. Lett. 86, 5675 (2001).

[7] R.M. Sternheimer, Phys. Rev. 115, 1198 (1959).

[8] A.D. Buckingham, Adv. Chem. Phys. 12, 107 (1967).

[9] A.J. Sadlej, Int. J. Quantum Chem. 23, 147 (1983).

[10] A.K. Bhatia and R.J. Drachman, Phys. Rev. A 59, 205 (1999).

[11] H. Hellmann, Einführung in die Quantenchemie (Deuticke, Leipzig, 1937), p. 285.

[12] R.P. Feynman, Phys. Rev. 56, 340 (1939).

[13] M. Cohen and G.W.F. Drake, Proc. Phys. Soc. London 92, 23 (1967).

[14] A. Dalgarno, Adv. Phys. 11, 281 (1962).

[15] R.M. Sternheimer, Phys. Rev. 96, 951 (1954).

[16] K. Singer, Proc. R. Soc. London, Ser. A 258, 412 (1960).

[17] W. Cencek and W. Kutzelnigg, J. Chem. Phys. 105, 5878 (1996).
[18] W. Cencek, J. Komasa, and J. Rychlewski, Chem. Phys. Lett. 246, 417 (1995).

[19] D.B. Kinghorn and L. Adamowicz, J. Chem. Phys. 113, 4203 (2000).

[20] J. Komasa, W. Cencek, and J. Rychlewski, in Computational Methods in Science and Technology (Scientific Publishers OWN, Poznań, 1996), Vol. 2, p. 87.

[21] J. Rychlewski, Adv. Quantum Chem. 31, 173 (1999).

[22] W. Cencek and J. Rychlewski, Chem. Phys. Lett. 320, 549 (2000).

[23] J. Komasa and J. Rychlewski, Mol. Phys. 91, 909 (1997).

[24] J. Komasa, J. Chem. Phys. 110, 7909 (1999).

[25] J. Komasa and J. Rychlewski, Pol. J. Chem. 72, 1353 (1998).

[26] J. Komasa, W. Cencek, and J. Rychlewski, Phys. Rev. A 52, 4500 (1995).

[27] J. Komasa and J. Rychlewski, Chem. Phys. Lett. 342, 185 (2001).

[28] K. Strasburger, J. Chem. Phys. 111, 10555 (1999).

[29] J. Komasa, J. Chem. Phys. 112, 7075 (2000).

[30] J. Komasa, J. Chem. Phys. 115, 158 (2001).

[31] J.O. Hirschfelder, W. Byers Brown, and S.T. Epstein, Adv. Quantum Chem. 1, 255 (1964).

[32] E. Hylleraas, Z. Phys. 65, 209 (1930). 
[33] S. T. Epstein, in The Variation Method in Quantum Chemistry, edited by E. M. Loebl (Academic Press, New York, 1974).

[34] H.J. Kolker and H.H. Michels, J. Chem. Phys. 43, 1027 (1965).

[35] R.M. Glover and F. Weinhold, J. Chem. Phys. 65, 4913 (1976).

[36] J.S. Sims and J.R. Rumble, Jr., Phys. Rev. A 8, 2231 (1973).

[37] J. Rychlewski, Mol. Phys. 41, 833 (1980).

[38] W. Kolos and L. Wolniewicz, J. Chem. Phys. 46, 1426 (1967).

[39] J. Rychlewski, Chem. Phys. Lett. 73, 135 (1980).

[40] J. Rychlewski, J. Chem. Phys. 78, 7252 (1983).

[41] J. Rychlewski, J. Chem. Phys. 81, 6007 (1984).

[42] J. Rychlewski, in Molecules in Physics, Chemistry, and Biology, edited by J. Maruani (Kluwer Academic Publishers, Dordrecht, 1988), Vol. II, p. 207.

[43] J. Rychlewski, Croat. Chem. Acta 61, 799 (1989).

[44] J. Rychlewski, J. Komasa, and W. Cencek, Phys. Rev. A 41, 5825 (1990).

[45] W. Cencek, J. Komasa, and J. Rychlewski, in Handbook on Parallel and Distributed Processing: High-Performance Computing in Molecular Sciences, edited by J. Blazewicz, K. Ecker, B. Plateau, and D. Trystram (Springer-Verlag, Berlin, 2000), p. 505.

[46] V.I. Korobov, Phys. Rev. A 61, 064503 (2000).

[47] G. W. F. Drake, in Long-Range Casimir Forces: Theory and Recent Experiments on Atomic Systems, edited by F. S. Levine, and D. A. Micha (Plenum Press, New York, 1993), p. 107.

[48] Z.-C. Yan, J.F. Babb, A. Dalgarno, and G.W.F. Drake, Phys. Rev. A 54, 2824 (1996).

[49] Z.-C. Yan, M. Tambasco, and G.W.F. Drake, Phys. Rev. A 57, 1652 (1998).

[50] Z.-C. Yan and G.W.F. Drake, Phys. Rev. A 52, R4316 (1995).

[51] G. Pestka and W. Woźnicki, Chem. Phys. Lett. 255, 281 (1996).

[52] T.P. Das and R. Bersohn, Phys. Rev. 102, 733 (1956).

[53] C. Schwartz, Ann. Phys. (N.Y.) 6, 170 (1959).

[54] W. Cencek (private communication).

[55] P.C. Mahapatra and B.K. Rao, Z. Phys. D: At., Mol. Clusters 16, 17 (1990).

[56] F. Weinhold, Proc. R. Soc. London, Ser. A 327, 209 (1972).

[57] F. Weinhold, Adv. Quantum Chem. 6, 299 (1972).

[58] C. Eckart, Phys. Rev. 36, 878 (1930).

[59] H.F. Weinberger, J. Res. Natl. Inst. Stand. Technol. 64, 217 (1960).
[60] F. Weinhold, J. Chem. Phys. 59, 355 (1973).

[61] NIST Atomic Spectra Database: http://physics.nist.gov/.

[62] D. Bégué, M. Merawa, and C. Pouchan, Phys. Rev. A 57, 2470 (1998).

[63] G. Maroulis and A.J. Thakkar, J. Phys. B 21, 3819 (1988).

[64] M.G. Papadopoulos, J. Waite, and A.D. Buckingham, J. Chem. Phys. 102, 371 (1995).

[65] D. Tunega, J. Noga, and W. Klopper, Chem. Phys. Lett. 269, 435 (1997).

[66] G. Figari, G.F. Musso, and V. Magnasco, Mol. Phys. 50, 1173 (1983).

[67] A.J. Thakkar, Phys. Rev. A 40, 1130 (1989).

[68] W.J. Stevens and F.P. Billingsley, Phys. Rev. A 8, 2236 (1973).

[69] H.-J. Werner and W. Meyer, Phys. Rev. A 13, 13 (1976).

[70] W. Müller, J. Flesch, and W. Meyer, J. Chem. Phys. 80, 3297 (1984).

[71] R.L. Graham and D.L. Yeager, Int. J. Quantum Chem. 31, 99 (1987).

[72] M. Urban, G.H.F. Diercksen, A.J. Sadlej, and J. Noga, Theor. Chim. Acta 77, 29 (1990).

[73] H. Koch and R.J. Harrison, J. Chem. Phys. 95, 7479 (1991).

[74] S.I. Themelis and C.A. Nicolaides, Phys. Rev. A 52, 2439 (1995).

[75] M.G. Papadopoulos and A.J. Sadlej, Chem. Phys. Lett. 288, 377 (1998).

[76] A.W.K. Leung and W.H. Breckenridge, J. Chem. Phys. 111, 9197 (1999).

[77] E.-A. Reinsch and W. Meyer, Phys. Rev. A 18, 1793 (1978).

[78] A. Dalgarno and J.M. McNamee, J. Chem. Phys. 35, 1517 (1961).

[79] M. Cohen, A. Dalgarno, and J.M. McNamee, Proc. R. Soc. London, Ser. A 269, 550 (1962).

[80] H.P. Kelly, Phys. Rev. 136, B896 (1964).

[81] V.G. Kaveeshwar, A. Dalgarno, and R.P. Hurst, J. Phys. B 2, 984 (1969).

[82] P.W. Fowler and A.D. Buckingham, Chem. Phys. 98, 167 (1985).

[83] D.M. Bishop and S.M. Cybulski, J. Chem. Phys. 93, 590 (1990).

[84] W.A. Parkinson, S.P.A. Sauer, J. Oddershede, and D.M. Bishop, J. Chem. Phys. 98, 487 (1993).

[85] P. Jørgensen, J. Oddershede, P. Albertsen, and N.H.F. Beebe, J. Chem. Phys. 68, 2533 (1978). 